\title{
GAIA Provider Initiated Preterm Delivery Levels of Diagnostic Certainty
}

National Cancer Institute

\section{Source}

National Cancer Institute. GAIA Provider Initiated Preterm Delivery Levels of Diagnostic

Certainty. NCl Thesaurus. Code C127943.

A classification of maternal and fetal outcomes relating to the assessment of provider initiated preterm delivery, developed by the Global Alignment of Immunization safety Assessment in pregnancy, based on the extent to which the diagnosis has been confirmed. 